\title{
Deteç̧ão de anticorpos anti-Rickettsia spp. em cães e equinos no estado de Mato Grosso, Brasil
}

\section{Detection of antibodies anti-Rickettsia spp. in dogs and horses in the state of Mato Grosso, Brazil}

\author{
Marcus Vinicius de Amorim ${ }^{1 *}$; Andréia Lima Tomé Melo \\ André Luis Santos de Freitas²; Ricardo Pereira Laub²; Rosane Marini Melo \\ Daniel Moura de Aguiar ${ }^{4}$; Anderson Castro Soares de Oliveira ${ }^{5}$; \\ Jonas Moraes-Filho ${ }^{6}$; Marcelo Bahia Labruna ${ }^{7}$ : Richard de Campos Pacheco ${ }^{4}$
}

\section{Resumo}

Este estudo avaliou a infecção causada por Rickettsia spp. em cães e equinos no Estado de Mato Grosso . Um total de 384 cães e 460 equinos foi testado pela reação de imunofluorescência indireta (RIFI) para Rickettsia rickettsii, Rickettsia parkeri, Rickettsia amblyommii, Rickettsia rhipicephali e Rickettsia bellii. No total, 3,12\% (12/384) dos cães foram soros reagentes para pelo menos uma espécie de Rickettsia. Um total de sete (1,82 \%) soros caninos apresentaram títulos para $R$. bellii, pelo menos 4 vezes maior do para qualquer um dos outros antígenos de riquétsia, permitindo considerar que estes cães foram infectados por $R$. bellii. Nos equinos, 273 (59,34 \%) foram positivos para pelo menos um antígeno testado, e os maiores títulos finais anti-Rickettsia spp. foram observados para R. amblyommii, sugerindo reações homólogas a este agente ou um organismo intimamente relacionado. Os resultados mostraram que os cães estão sob baixa exposição a carrapatos infectados com riquétsias do grupo da febre maculosa, indicando baixo risco de infecção humana por esses agentes. No entanto, R. amblyommii está amplamente distribuída no Estado.

Palavras-chave: Equinos e cães, reação de imunofluorescência indireta, soroprevalência, Rickettsia spp., Mato Grosso

\footnotetext{
Abstract

This study evaluated the infection caused by Rickettsia spp. among dogs and horses from Mato Grosso State. A total of $384 \mathrm{dogs}$ and 460 horses were tested by the Indirect Immunofluorescence Assay (IFA) for Rickettsia rickettsii, Rickettsia parkeri, Rickettsia amblyommii, Rickettsia rhipicephali and Rickettsia bellii Overall, 3.12\% (12/384) of the dogs were seroreactive for at least one Rickettsia

${ }^{1}$ Discentes do Programa de Pós-graduação em Ciências Veterinária da Universidade Federal de Mato Grosso, UFMT, Cuiabá, MT. E-mail: marviamorim@gmail.com; andreialtm@gmail.com

${ }^{2}$ Discentes do Curso de Medicina Veterinária da UFMT, Cuiabá, MT. E-mail: andreyagami@hotmail.com; ricardolaub@gmail.com

${ }^{3}$ Pesquisadora do Instituto de DefesaAgropecuário do Estado de Mato Grosso, INDEA-MT, Cuiabá, MT. E-mail:rosanemarinimelo@

${ }^{4}$ Profs. da Faculdade de Agronomia, Medicina Veterinária e Zootecnia, UFMT, Cuiabá, MT. E-mail: danmoura@ufmt.br; richard@

${ }^{5}$ Prof. do Instituto de Ciências Exatas e da Terra, UFMT, Cuiabá, MT. E-mail: andersoncso@ufmt.br

${ }^{6}$ Discente do Programa de Pós-graduação em Epidemiologia Experimental Aplicada às Zoonoses, Universidade de São Paulo, USP, São Paulo, SP. E-mail: jonasmfilho@usp.br

${ }^{7}$ Prof. da Faculdade de Medicina Veterinária e Zootecnia, USP, São Paulo, SP. E-mail: labruna@usp.br

* Autor para correspondência
} gmail.com ufmt.br 
species. A total of 7 (1.82\%) canine sera showed titers to $R$. bellii at least 4-fold higher than those to any of the other rickettsial antigens, allowing us to consider that these dogs were infected by $R$. bellii. In horses, $273(59.34 \%$ ) samples were positives for at least one antigen tested, and highest anti-Rickettsia spp. endpoint titers were observed for $R$. amblyommii, suggesting homologous reactions to this agent or a very closely related organism. The results showed that dogs are under low exposition to ticks infected with spotted fever group Rickettsia, indicating low risk of human infection by these agents. However, $R$. amblyommii is widely distributed in the State.

Key words: Horses and dogs, indirect immunofluorescence assay, seroprevalence, Rickettsia spp., Mato Grosso

\section{Introdução}

Rickettsia é uma bactéria intracelular obrigatória, pertencente à família Rickettsiaceae e ordem Rickettsiales (RAOULT; ROUX, 1997). As espécies patogênicas deste gênero estão divididas em dois grupos: i-grupo Tifo (GT), com a Rickettsia prowazekii e Rickettsia typhi (EREMEEVA; DASCH, 2000); ii-grupo da Febre Maculosa (GFM). Há também duas espécies de patogenicidade desconhecida, Rickettsia canadensis e Rickettsia bellii, que não estão inseridas em nenhum destes grupos (LABRUNA et al., 2011).

No GFM, há mais de 20 espécies válidas, dentre elas algumas descritas no Brasil, como a Rickettsia rickettsii, Rickettsia parkeri e Rickettsia felis, patogênicas aos humanos, enquanto a Rickettsia rhipicephali, Rickettsia amblyommii apresentam patogenicidade desconhecida. Há outras espécies de riquétsias descritas no país, como a $R$. typhi, R. bellii e Rickettsia monteiroi (LABRUNA et al., 2011).

A $R$. rickettsii é considerada a espécie mais patogênica dentre as descritas no mundo (PAROLA; PADDOCK; RAOULT, 2005), sendo responsável por causar a doença denominada de Febre Maculosa das Montanhas Rochosas (FMMR) nos Estados Unidos. As diversas espécies de riquétsias têm, em sua maioria, os carrapatos como principal vetor, com exceção da $R$. felis e $R$. typhi, veiculadas por pulgas, e da $R$. prowazekii e Rickettsia akari, transmitidas por piolhos e ácaros, respectivamente (RAOULT; ROUX, 1997; LABRUNA, 2009).

No Brasil, a infecção por $R$. rickettsii é conhecida como Febre Maculosa Brasileira (FMB), e sua transmissão tem sido associada, principalmente aos carrapatos Amblyomma cajennense e Amblyomma aureolatum, sendo o Rhipicephalus sanguineus considerado um provável vetor na região metropolitana de São Paulo (LABRUNA, 2009).

O uso de técnicas sorológicas, principalmente a Reação de Imunofluorescência Indireta (RIFI), na detecção de anticorpos anti-Rickettsia spp. em animais (equinos e/ou cães) já foi descrito em estudos epidemiológicos nos estados de Rondônia (LABRUNA et al., 2007b), Mato Grosso (MELO et al., 2011), São Paulo (HORTA et al., 2007; PINTER et al., 2008; MORAES-FILHO et al., 2009), Minas Gerais (VIANNA et al., 2008; SILVA et al., 2010; PACHECO et al., 2011), Espírito Santo (SPOLIDORIO et al., 2010b), Paraná (TAMEKUNI et al., 2011; TOLEDO et al., 2011), Santa Catarina (MEDEIROS et al., 2013) e Rio Grande do Sul (SAITO et al., 2008; SANGIONI et al., 2011).

Os equinos, abundantes em muitas áreas endêmicas para FMB, juntamente com os cães, são considerados animais sentinelas da doença, por servirem como hospedeiros primários aos carrapatos vetores (HORTA et al., 2004; SANGIONI et al., 2005; LABRUNA, 2009). No entanto, a circulação e manutenção das riquétsias dependem de hospedeiros amplificadores, como os gambás (Didelphis marsupialis) e as capivaras (Hydrochoerus hydrochaeris), que mantêm níveis altos de bacteremia por um período prolongado, permitindo que carrapatos não infectados adquiram a infecção (BURGDORFER, 1988; HORTA et al., 2009; SOUZA et al., 2009). As infecções por riquétsias em capivaras (PACHECO et al., 2007), 
gambás (HORTA et al., 2007) e pequenos mamíferos não voadores (PENA et al., 2009; MILAGRES et al., 2013), também vêm sendo descritas por meio de análises sorológicas.

Melo et al. (2011) observaram pela RIFI uma prevalência de $47,5 \%$ de anticorpos anti-Rickettsia spp. contra pelo menos um dos antígenos testados nos cães do município de Poconé, região do Pantanal mato-grossense, sendo $R$. amblyommii a espécie predominante. Considerando que $R$. amblyommii está associada como possível causadora de doença febril em humanos nos Estados Unidos (APPERSON et al., 2008), e da provável importância do agente em saúde pública, bem como a deficiência de estudos acerca das riquétsias no Estado, este trabalho objetivou detectar a presença de anticorpos anti-Rickettsia spp. em animais sentinelas (equinos e cães), com intuito de determinar a possível circulação dos agentes, elucidando melhor a sua distribuição e permitindo ações estratégicas para estudos futuros.

\section{Material e Métodos}

Área de estudo

O Estado do Mato Grosso está localizado na região Centro-Oeste do Brasil, tendo 141 municípios em sua composição, sendo Cuiabá a capital. O território ocupa uma área de 903.366,192 $\mathrm{km}^{2}$, tendo uma população estimada de 3,1 milhões de habitantes (IBGE, 2012). Situado no centro geodésico da América Latina, Mato Grosso apresenta predominantemente o clima tropical super-úmido, com alta pluviosidade e média de temperatura superior a $24^{\circ} \mathrm{C}$, além do clima tropical com chuvas de verão e inverno seco. A região apresenta uma rica fauna e flora, contemplada pelos seus três biomas: o cerrado, considerado a savana brasileira, com mais de 1.500 espécies de seres vertebrados e invertebrados descritos; o pantanal, a maior área alagável e uma das faunas mais ricas do planeta; e a Amazônia, considerada uma das mais complexas biodiversidades no mundo, devido à abundância e grossuras das copas, que dificulta o acesso de luminosidade na floresta (MTGOV, 2012).

\section{Amostras}

Foram coletadas 384 amostras de sangue de cães (utilizando taxa de prevalência de $50 \%$, erro estatístico de $5 \%$ e intervalo de confiança de $95 \%$ ), acondicionadas em tubos com EDTA, atendidos no Hospital Veterinário da Universidade Federal de Mato Grosso (HOVET-UFMT), campus Cuiabá, entre o período de Agosto de 2011 e Julho de 2012, sendo todos os animais provenientes da área urbana (dados não publicados). Após a centrifugação (1500 $\mathrm{x}$ g por 10 minutos) do material, o plasma obtido foi identificado e armazenado a $20{ }^{\circ} \mathrm{C}$ negativos até o momento do processamento. $\mathrm{O}$ sangue coletado foi submetido às análises hematológicas, obtendo-se valores relacionados ao hematócrito, hemoglobina, eritrócitos e plaquetas (MEINKOTH; CLINKENBEARD, 2000). Informações referentes à idade do paciente, exposição ao carrapato ou histórico de doenças transmitidas por esse vetor foram reunidas através da ficha de anamnese do animal.

Para as amostras de soro dos equinos, o estado foi divido em 12 unidades regionais (Figura 1), segundo o mapa do Instituto de Defesa Agropecuária do Estado do Mato Grosso (INDEA-MT). Realizou-se o cálculo estratificado da população total de equinos de cada regional, observando dados do INDEAMT, utilizando prevalência de $50 \%$, intervalo de confiança de $95 \%$, e erro padrão de $5 \%$, perfazendo um total de 400 amostras. No entanto, foi acrescido um número maior de soros à amostragem das regionais de Alta Floresta, Rondonópolis, Lucas do Rio Verde e Sinop, com a finalidade de tornar mais precisa a representação nessas localidades, totalizando 460 animais (Figura 1). As amostras foram cedidas pelo Laboratório de Sanidade Animal (LASA) do INDEA-MT, previamente enviadas para realização do diagnóstico de Anemia Infecciosa Equina (AIE). 
Figura 1. Distribuição do número de amostras de soro equino e das espécies de riquétsias determinadas como provável antígeno responsável pela infecção (PARI) pela reação de imunofluorescência indireta (RIFI) nas 12 unidades regionais, segundo o mapa do Instituto de Defesa Agropecuária do Estado do Mato Grosso (INDEA-MT), 2012.
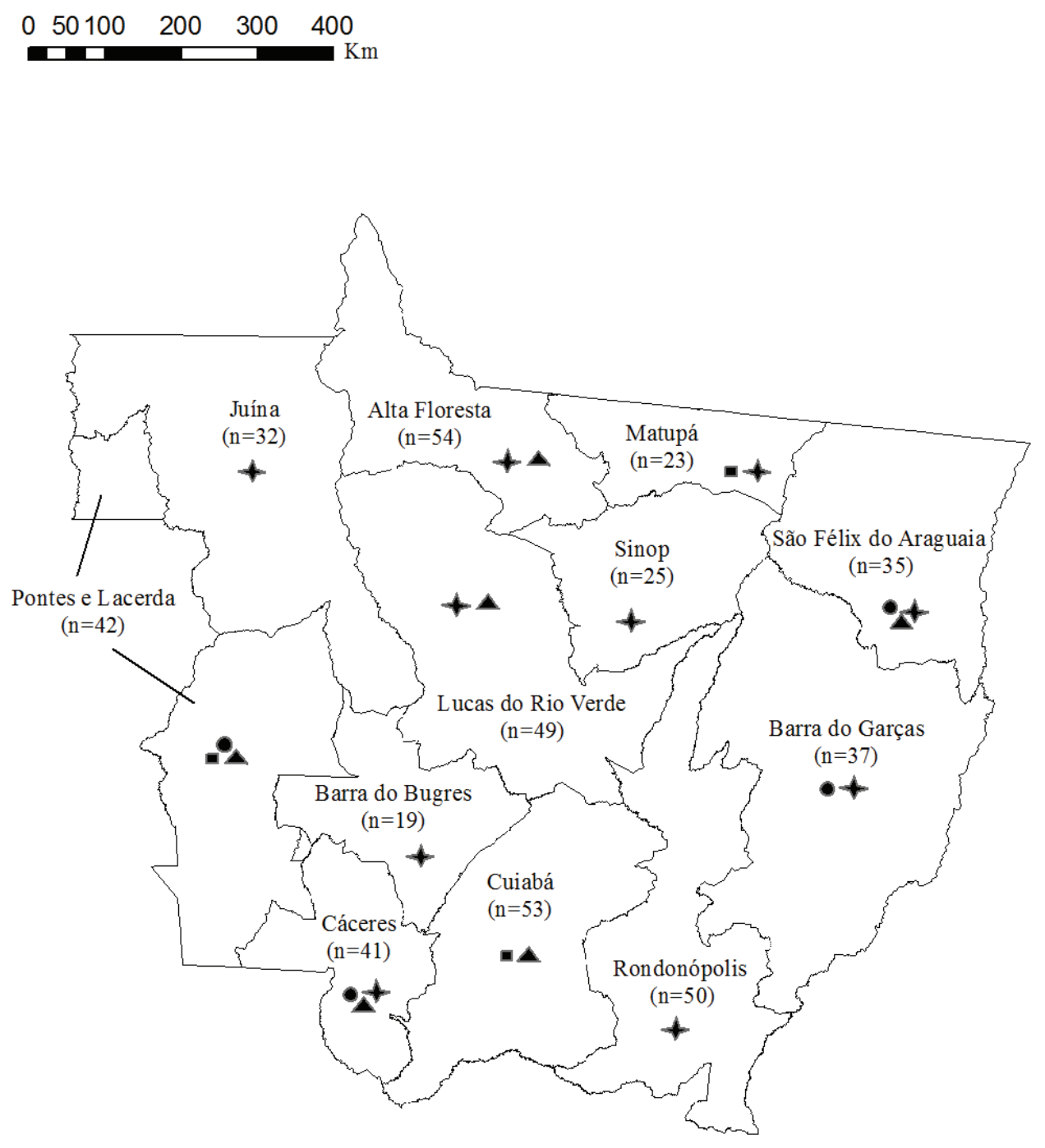

\section{LEGENDA:}

PARI (provável antíg eno responsável pela infecção):

+ Rickettsia amblyommii

A Rickettsia bellii

- Rickettsia rickettsii

- Rickettsia rhipicephali

n: número de animais amostrados

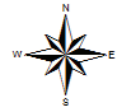

Fonte: Elaboração dos autores. 


\section{Cultivo celular}

A produção dos inóculos e confecção das lâminas foram realizadas, segundo Horta et al. (2004), utilizando como antígenos brutos as espécies $R$. rickettsii cepa Taiaçu e $R$. bellii cepa Mogi (PINTER; LABRUNA, 2006), $R$. parkeri cepa At24 (SILVEIRA et al., 2007), R. amblyommii cepa Ac37 (LABRUNA et al., 2004) e $R$. rhipicephali cepa HJ5 (LABRUNA et al., 2007a).

\section{Reação de Imunofluorescência Indireta (RIFI)}

A RIFI foi realizada conforme Horta et al. (2007), sendo as amostras de plasma dos cães e de soros dos equinos diluídas a 1:64 em Solução Tampão Fosfatada (PBS), pH 7,2 (0,0084M Na $\mathrm{HPO}_{4}$, $0,0018 \mathrm{M} \mathrm{NaH}_{2} \mathrm{PO}_{4}$ e $\left.0,147 \mathrm{M} \mathrm{NaCl}\right)$ e aplicadas às laminas com antígeno fixado e incubadas por 30 minutos à $37^{\circ} \mathrm{C}$ em câmara úmida. Em seguida, foram realizadas duas lavagens de 10 minutos em PBS e após a secagem das mesmas, foi adicionado conjugado de coelho anti-IgG de cão e equino (Sigma Diagnostics, St. Luis, Mo), segundo cada espécie animal, na diluição 1:800 e 1:1000, respectivamente. Novamente, as lâminas foram incubadas a $37{ }^{\circ} \mathrm{C}$ por 30 minutos e lavadas duas vezes de 10 minutos em PBS acrescido com Azul de Evans. Após a secagem das lâminas, foi aplicada glicerina, $\mathrm{pH}$ 8,5 e estas foram examinadas em microscópio (ZEISS AX10) de epifluorescência, com objetiva de 40x. As amostras consideradas positivas (título $\geq 64$ ) foram sucessivamente diluídas na razão dois para obtenção do título final. Quando um título obtido para uma determinada espécie de riquétsia foi pelo menos quatro vezes maior do que o título mais alto obtido para as demais espécies, pode-se sugerir que os anticorpos foram homólogos à primeira espécie, sendo considerado o provável antígeno responsável pela infecção (PARI) (LABRUNA et al., 2007b). Em cada lâmina foram adicionados soros-controle sabidamente positivo e negativo.

Para análise estatística das variáveis qualitativas (sexo, idade, exposição ao vetor, alteração hematológica, período do ano) foi utilizado o teste Qui-Quadrado $\left(x^{2}\right)$ através do programa Epi Info ${ }^{\mathrm{TM}}$ 7.

O projeto de pesquisa foi aprovado pelo Comitê de Ética no Uso de Animais em Pesquisa (CEUA) da UFMT (Protocolo n. ${ }^{\circ}$ 23108.003.203/11-9).

\section{Resultados}

Dos 384 cães amostrados, 200 (52,08\%) eram fêmeas e 184 (47,92\%) eram machos, 89 (23,18\%) tinham idade inferior a um ano e 295 (76,82\%) com idade superior ou igual a um ano. Em relação às alterações hematológicas: 89(23,18\%) apresentavam anemia; $58(15,10 \%)$ trombocitopenia; 109 $(28,39 \%)$ anemia e trombocitopenia e $128(33,33 \%)$ apresentavam valores dentro dos padrões normais. Dos animais estudados, 130 (33,85\%) tiveram contato com carrapatos ou manifestaram alguma doença transmitida por carrapatos e $254(66,15 \%)$ não tiveram contato com este vetor ou não foi possível obter esta informação na ficha de anamnese. Das amostras submetidas à RIFI, 11 (2,86\%) foram reagentes (título $\geq 64$ ) para pelo menos um antígeno testado e $373(97,14 \%)$ soronegativos. Os cães reagentes mostraram títulos que variaram de 64 a 2048 para pelo menos um dos cinco antígenos (Tabela 1), sendo que em sete $(63,6 \%)$ amostras determinou-se a $R$. bellii como PARI.

Das 460 amostras de soro de equino submetidas à RIFI, foram observados animais reagentes (título $\geq 64$ ) nas 12 unidades regionais, com porcentagem de soropositividade que variaram de 16\% (Rondonópolis) a 82,60\% (Matupá), conforme Tabela 2, com títulos de anticorpos que variaram de 64 a 2048. R. amblyommii foi considerada como PARI em soros de 131 equinos, englobando 10 $(83,34 \%)$ das 12 regionais avaliadas, exceto nas regionais de Pontes e Lacerda e Cuiabá. $R$. bellii, $R$. rickettsii e $R$. rhipicephali foram incriminadas como PARI em amostras de soro de seis (50\%), quatro $(33,34 \%)$ e três $(25 \%)$ unidades regionais do Estado, respectivamente (Figura 1). 
Tabela 1. Títulos apresentados pela Reação de Imunofluorescência Indireta (RIFI) para cinco antígenos brutos de riquétsia em amostras de plasma de cães atendidos no HOVET-UFMT, Cuiabá-MT, 2011 a 2012.

\begin{tabular}{lcccccc}
\hline \multirow{2}{*}{$\begin{array}{c}\text { Plasma } \\
\text { canino }\end{array}$} & \multicolumn{5}{c}{ Título pela RIFI } \\
\cline { 2 - 5 } & $\begin{array}{c}\text { Rickettsia } \\
\text { rickettsii }\end{array}$ & $\begin{array}{c}\text { Rickettsia } \\
\text { parkeri }\end{array}$ & $\begin{array}{c}\text { Rickettsia } \\
\text { amblyommii }\end{array}$ & $\begin{array}{c}\text { Rickettsia } \\
\text { rhipicephali }\end{array}$ & $\begin{array}{c}\text { Rickettsia } \\
\text { bellii }\end{array}$ & \multirow{2}{*}{ PARI } \\
\hline N10 & NR & NR & 1024 & 2048 & NR & ND \\
N113 & NR & NR & NR & NR & 1024 & R. bellii \\
N132 & NR & NR & NR & NR & 1024 & R. bellii \\
N146 & NR & NR & NR & NR & 2048 & R. bellii \\
N223 & NR & NR & NR & NR & 2048 & R. bellii \\
N227 & NR & NR & NR & NR & 512 & R. bellii \\
N269 & NR & NR & NR & NR & 1024 & R. bellii \\
N285 & NR & NR & NR & NR & 128 & R. bellii \\
N350 & NR & NR & NR & NR & 64 & ND \\
N364 & 1024 & 512 & 512 & NR & NR & ND \\
N366 & 128 & 256 & 256 & NR & NR & ND \\
\hline
\end{tabular}

PARI: provável antígeno responsável pela infecção; NR: soro não reagente a um título $\geq 64$; ND: não determinado

Fonte: Elaboração dos autores.

Tabela 2. Soroprevalência pela Reação de Imunofluorescência Indireta (RIFI) para antígenos de cinco espécies de riquétsias em soro de equinos das 12 unidades regionais, segundo o mapa do Instituto de Defesa Agropecuária do Estado do Mato Grosso (INDEA-MT), 2012.

\begin{tabular}{|c|c|c|c|c|c|c|c|c|}
\hline \multirow[b]{2}{*}{ Regional } & \multirow[b]{2}{*}{$\mathrm{n}$} & \multirow[b]{2}{*}{$\begin{array}{c}\mathrm{n} .^{\circ} \\
\text { positivos }\end{array}$} & \multirow[b]{2}{*}{$\%$} & \multicolumn{5}{|c|}{ PARI } \\
\hline & & & & $\begin{array}{c}\text { Rickettsia } \\
\text { rickettsii }\end{array}$ & $\begin{array}{l}\text { Rickettsia } \\
\text { parkeri }\end{array}$ & $\begin{array}{l}\text { Rickettsia } \\
\text { amblyommii }\end{array}$ & $\begin{array}{l}\text { Rickettsia } \\
\text { rhipicephali }\end{array}$ & $\begin{array}{c}\text { Rickettsia } \\
\text { bellii }\end{array}$ \\
\hline 1- Alta Floresta & 54 & 43 & $79.62 \%$ & ND & ND & 20 & ND & 1 \\
\hline 2- Barra do Bugres & 19 & 11 & $57.89 \%$ & ND & ND & 8 & ND & ND \\
\hline 3- Barra do Garças & 37 & 22 & $59.45 \%$ & 2 & ND & 13 & ND & ND \\
\hline 4- Cáceres & 41 & 33 & $80.48 \%$ & 2 & ND & 6 & ND & 4 \\
\hline 5- Cuiabá & 53 & 28 & $52.83 \%$ & ND & ND & 12 & 2 & 4 \\
\hline 6- Juína & 32 & 25 & $78.12 \%$ & ND & ND & 20 & ND & ND \\
\hline $\begin{array}{l}\text { 7- Lucas do Rio } \\
\text { Verde }\end{array}$ & 49 & 23 & $46.93 \%$ & ND & ND & 19 & ND & 1 \\
\hline 8- Matupá & 23 & 19 & $82.60 \%$ & ND & ND & 4 & 1 & ND \\
\hline 9- Pontes e Lacerda & 42 & 18 & $42.85 \%$ & 1 & ND & ND & 2 & 2 \\
\hline 10- Rondonópolis & 50 & 8 & $16.00 \%$ & ND & ND & 4 & ND & ND \\
\hline $\begin{array}{l}\text { 11- São Félix do } \\
\text { Araguaia }\end{array}$ & 35 & 23 & $65.71 \%$ & 1 & ND & 17 & ND & 1 \\
\hline 12-Sinop & 25 & 20 & $80 \%$ & ND & ND & 8 & ND & ND \\
\hline Total & 460 & 273 & $59.34 \%$ & 6 & 0 & 131 & 5 & 13 \\
\hline
\end{tabular}

$\mathrm{n}=$ número de amostras por regional; $n .^{\circ}$ positivos: número de amostras de soro equino positivas (título $\geq 64$ );

\%: percentual de amostras de soro positivos por unidade regional; PARI: provável antígeno responsável pela infecção;

ND: não determinado.

Fonte: Elaboração dos autores.

\section{Discussão e Conclusões}

A sorologia realizada em cães revelou menor prevalência de anticorpos para Rickettsia spp.
$(2,86 \%)$, quando comparada com estudos referentes à detecção de anticorpos em cães provenientes de áreas endêmicas para FMB nos 
estados de São Paulo e Minas Gerais, onde o percentual de animais soropositivos foi de até 64 e 67,9\%, respectivamente (PINTER et al., 2008; PACHECO et al., 2011). Esses dados confirmam a característica do município de área não endêmica para FMB ou qualquer outra espécie de riquétsia do GFM, assim como observados por Silva et al. (2010) em Minas Gerais e Fortes et al. (2010) no Paraná que encontraram, respectivamente, valores de 0,66 e $4,4 \%$.

A circulação de riquétsias na natureza é um processo dinâmico que envolve a participação de diferentes espécies de carrapatos e animais vertebrados, de maneira que a vigilância epidemiológica ativa deve ser adotada continuamente, a fim de determinar o surgimento de novas áreas endêmicas para riquetsioses, mesmo antes da ocorrência de casos humanos da doença (SILVA et al., 2010).

Melo et al. (2011) em um estudo no município de Poconé, localizado do pantanal mato-grossense, encontraram um panorama diferente do presente estudo, mostrando esse processo dinâmico das infecções riquetsiais. Os autores observaram uma taxa de prevalência de 19,3\% de cães soropositivos para Rickettsia spp. na área urbana, significativamente menor do que a observada nos animais provenientes da área rural $(75,6 \%)$. Esta maior prevalência estaria associada a maior chance dos cães daquela localidade estarem expostos à infestação pelo carrapato vetor (Amblyomma spp.), pois mesmo na área urbana, teriam acesso a regiões de mata, diferentemente dos cães do presente estudo, os quais eram provenientes essencialmente da cidade, e estariam expostos a maior infestação pelo $R$. sanguineus (dados não publicados). Esse fato, associado às informações da baixa exposição aos carrapatos, segundo informações dos proprietários, poderiam colaborar também com a baixa prevalência observada.

Dos antígenos testados, somente $R$. bellii foi considerada como PARI (Tabela 1), mesmo sendo observado títulos para as demais espécies. A $R$. rhipicephali e a $R$. bellii apresentam patogenicidade desconhecida, apesar de Apperson et al. (2008) apontarem a $R$. amblyommii como agente provável causador de doença febril em humanos nos Estados Unidos. A R. rickettsii é considerada como a espécie mais patogênica entre as descritas no mundo (PAROLA; PADDOCK; RAOULT, 2005), sendo responsável por causar uma doença de elevada letalidade em São Paulo (ANGERAMI et al., 2006). No Brasil, a $R$. parkeri já foi isolada de diferentes espécies de carrapatos do gênero Amblyomma (LABRUNA et al., 2011), sendo associada à infecção em humano nos estados de São Paulo (SPOLIDORIO et al., 2010a) e da Bahia (SILVA et al., 2011). Apesar da R. bellii ser a mais comumente encontrada em carrapatos na América, (PHILIP et al., 1983) e no Brasil estar associada, principalmente a carrapatos do gênero Amblyomma (LABRUNA et al., 2004; PINTER; LABRUNA, 2006; LABRUNA et al., 2007a), com taxas de infecção variáveis (0$100 \%$ ), não há nenhum evidência direta ou indireta de infecção humana por este antígeno (PACHECO et al., 2009).

Em animais há evidência sorológica de infecção por $R$. bellii em capivaras do estado de São Paulo, possivelmente transmitidos por Amblyomma dubitatum (PACHECO et al., 2007), além de cães no Paraná (FORTES et al., 2010), Mato Grosso (MELO et al., 2011) e Pará (SPOLIDORIO et al., 2013) . Apesar disso, não há comprovação experimental da capacidade vetorial de nenhuma espécie de carrapato na transmissão de $R$. bellii para animais. Horta et al. (2010) observaram a soroconversão de gambás inoculados experimentalmente com $R$. bellii; entretanto, esses animais não apresentaram níveis de riquetsemia suficientes para infectar os carrapatos. Pinter et al. (2008), verificando a ocorrência de anticorpos contra Rickettsia spp., não observaram nenhum animal reativo a $R$. bellii, apesar da taxa de infecção nos carrapatos Amblyomma aureolatum coletados sobre os cães ser de 1,5\%.

Em relação à população de equinos, a determinação da R. amblyommii como PARI em 10 
unidades regionais mostraa ampla distribuição dessa bactéria pelo Estado, além da primeira evidência sorológica da infecção por $R$. amblyommii nessa espécie animal no Brasil. Não há comprovação do possível vetor para esta espécie de riquétsia, mas algumas evidências sugerem a participação do $A$. cajennense, pois os equinos são hospedeiros primários paras todos os estágios evolutivos desse carrapato (LABRUNA, 2009). Além disso, a $R$. amblyommii já foi isolada nessa espécie de carrapato em Rondônia (LABRUNA et al., 2004), além da detecção molecular em carrapatos $A$. cajennense do Pantanal mato-grossense (ALVES et al., 2013). Bermúdez et al. (2011) relataram uma forte interação entre o $A$. cajennense e a $R$. amblyommii, onde foi determinada a sua ocorrência pela PCR em carrapatos coletados de cães e equinos, assim como pela sorologia, onde a mesma foi incriminada como PARI. Além disso, Melo et al. (2011) observaram que os cães expostos ao $A$. cajennense no pantanal de Poconé, Mato Grosso apresentaram a probabilidade 5,9 vezes maior de estarem infectados por R. amblyommii. No entanto, o papel dos equinos como possível amplificador da bactéria para os carrapatos ainda precisa ser comprovado, o que contribuiria para a manutenção da bactéria em carrapatos $A$. cajennense na natureza.

Apesar do fato de haver animais com títulos de anticorpos homólogos à $R$. rickettsii, e dessa espécie apresentar alta letalidade aos seres humanos, esses dados devem ser observados com cautela, pois não há confirmação de casos de FMB no Estado do Mato Grosso.

\section{Agradecimentos}

Ao INDEA-MT, Conselho Nacional de Desenvolvimento Científico e Tecnológico-CNPq (Processo no: 476757/2010-0) e Fundação de Amparo à Pesquisa do Estado do Mato GrossoFAPEMAT (Processo $n^{\circ}$ : 285929/2010) pelo suporte financeiro.

\section{Referências}

ALVES, A. S.; MELO, A. L. T.; MARTINS, T. F.; MARCILI, A.; SOARES, H. S.; AMORIM, A. V.; LABRUNA, M. B.; AGUIAR, D. M.; PACHECO, R. C. Detecção molecular de Rickettsia amblyommii em carrapatos Amblyomma cajennense do Pantanal Matogrossense. In: INTERNATIONAL SYMPOSIUM ON TICK CONTROL AND TICK-BORNE DISEASES, 1., 2013, Campo Grande. Anais... Campo Grande: Embrapa Gado de Corte, 2013, p. 12-13.

ANGERAMI, R. N.; RESENDE, M. R.; FELTRIN, A. F. C.; KATZ, G.; NASCIMENTO, E. M.; STUCCHI, R. S. B.; SILVA, L. J. Brazilian spotted fever: a case series from an endemic area in Southeastern Brazil. Annals of the New York Academy of Sciences, New York, v. 1078, n. 1, p. 252-254, 2006.

APPERSON, C. S.; ENGBER, B.; NICHOLSON, W. L.; MEAD, D. G.; ENGEL, J.; YABSLEY, M. J.; DAIL, K.; JOHNSON, J.; WATSON, D. W. Tick-borne diseases in North Carolina: is "Rickettsia amblyommii" a possible cause of rickettsiosis reported as Rocky Mountain spotted fever? Vector-Borne and Zoonotic Diseases, Larchmont, v. 8, n. 5, p. 597-606, 2008.

BERMÚDEZ, C. S. E.; ZALDÍVAR, A. Y.; SPOLIDORIO, M. G.; MORAES-FILHO, J.; MIRANDA, R. J.; CABALLERO, C. M.; MENDOZA, Y.; LABRUNA, M. B. Rickettsial infection in domestic mammals and their ectoparasites in El Valle de Antón, Coclé, Panamá. Veterinary Parasitology, Amsterdam, v. 177, n. 1, p. 134-138, 2011.

BURGDORFER, W. Ecological and epidemiological considerations of Rock Mountain spotted fever and scrub typhus. In: WALKER, D. H. Biology of rickettsial diseases. Boca Raton: CRC, 1988. v. 1, p. 33-50.

EREMEEVA, M. E.; DASCH, G. A. Rickettsiae. In: LEDERBERG, J. (Ed.). Encyclopedia of microbialogy. New York: Academic Press, v. 4, 2000. p.140-180.

FORTES, F. S.; SILVEIRA, I.; MORAES-FILHO, J.; LEITE, R. V.; BONACIM, J. E.; BIONDO, A. W.; LABRUNA, M. B.; MOLENTO, M. B. Seroprevalence of Rickettsia bellii and Rickettsia felis in dogs, São José dos Pinhais, State of Paraná, Brazil. Revista Brasileira de Parasitologia Veterinária, Jaboticabal, v. 19, n. 4, p. 222-227, 2010.

HORTA, M. C.; LABRUNA, M. B.; SANGIONI, L. A.; VIANNA, M. C.; GENNARI, S. M.; GALVAO, M. A.; MAFRA, C. L.; VIDOTTO, O.; SCHUMAKER, T. T.; WALKER, D. H. Prevalence of antibodies to spotted fever group rickettsiae in humans and domestic animals in a Brazilian spotted fever-endemic area in the state 
of Sao Paulo, Brazil: serologic evidence for infection by Rickettsia rickettsii and another spotted fever group Rickettsia. The American Journal of Tropical Medicine and Hygiene, Deerfield, v. 71, n. 1, p. 93-97, 2004.

HORTA, M. C.; LABRUNA, M. B.; PINTER, A.; LINARDI, P. M.; SCHUMAKER, T. T. S. Rickettsia infection in five areas of the state of São Paulo, Brazil. Memorias do Instituto Oswaldo Cruz, Rio de Janeiro, v. 102, n. 7, p. 793-801, 2007.

HORTA, M. C.; MORAES-FILHO, J.; CASAGRANDE, R. A.; SAITO, T. B.; ROSA, S. C.; OGRZEWALSKA, M.; MATUSHIMA, E. R.; LABRUNA, M. B. Experimental infection of opossums Didelphis aurita by Rickettsia rickettsii and evaluation of the transmission of the infection to ticks Amblyomma cajennense. VectorBorne and Zoonotic Diseases, Larchmont, v. 9, n. 1, p. 109-118, 2009.

HORTA, M. C.; SABATINI, G. S.; MORAES FILHO, J.; OGRZEWALSKA, M.; CANAL, R. B.; PACHECO, R. C.; MARTINS, T. F.; MATUSHIMA, E. R.; LABRUNA, M. B. Experimental Infection of the Opossum Didelphis aurita by Rickettsia felis, Rickettsia bellii, and Rickettsia parkeri and Evaluation of the Transmission of the Infection to Ticks Amblyomma cajennense and Amblyomma dubitatum. Vector Borne and Zoonotic Diseases, Larchmont, v. 10, n. 10, p. 959-967, 2010.

INSTITUTO BRASILEIRO DE GEOGRAFIA E ESTATÍSTICA - IBGE. Unidades da federação, estados, Mato Grosso. Rio de Janeiro: IBGE, 2012. Disponível em: <http://www.ibge.gov.br>. Acesso em: 01 dez. 2012.

LABRUNA, M. B.; WHITWORTH, T.; BOUYER, D. H.; MCBRIDE, J.; CAMARGO, L. M. A.; CAMARGO, E. P.; POPOV, V.; WALKER, D. H. Rickettsia bellii and Rickettsia amblyommii in Amblyomma ticks from the State of Rondonia, Western Amazon, Brazil. Journal of Medical Entomology, Annapolis, v. 41, n. 6, p. 10731081, 2004.

LABRUNA, M.B.; PACHECO, R.C.; RICHTZENHAIN, L. J.; SZABÓ, M. P. J. Isolation of Rickettsia rhipicephali and Rickettsia bellii from Haemaphysalis juxtakochi ticks in the state of São Paulo, Brazil. Applied and Environmental Microbiology, Washington D.C., v. 73, n. 3, p. 869-873, 2007a.

LABRUNA, M. B.; HORTA, M. C.; AGUIAR, D. M.; CAVALCANTE, G. T.; PINTER, A.; GENNARI, S. M.; CAMARGO, L. M. A. Prevalence of Rickettsia infection in dogs from the urban and rural areas of Monte Negro Municipality, western Amazon, Brazil. Vector-Borne and Zoonotic Diseases, Larchmont, v. 7, n. 2, p. 249-255, $2007 b$.
LABRUNA, M. B. Ecology of Rickettsia in South America. Annals of the New York Academy of Sciences, New York, v. 1166, n. 1, p. 156-166, 2009.

LABRUNA, M. B.; MATTAR, V.; NAVA, S.; BERMUDEZ, S.; VENZAL, J. M.; DOLZ, G.; ABARCA, K.; ROMERO, L.; DE SOUSA, R.; OTEO, J. Rickettsioses in Latin America, Caribbean, Spain and Portugal. Revista MVZ Córdoba, Montería, v. 16, n. 2, p. 2435-2457, 2011.

MEDEIROS, A. P.; MOURA, A. B.; SOUZA, A. P.; BELLATO, V.; SARTOR, A. A.; VIEIRA-NETO, A.; MORAES-FILHO, J.; LABRUNA, M. B. Antibodies against rickettsiae from spotted fever groups in horses from two mesoregions in the state of Santa Catarina, Brazil. Arquivo Brasileiro de Medicina Veterinária e Zootecnia, Belo Horizonte, v. 65, p. 1713-1719, 2013.

MEINKOTH, J. H.; CLINKENBEARD, K. D. Normal hematology of the dog. In: FELDMAN, B.F.; ZINKL, J. G.; JAIN, N. C. Schaml's veterinary hematology. 5. ed. Filadélfia: Lippincott Willians \& Wilkins, 2000. p. 10571063.

MELO, A. L. T.; MARTINS, T. F.; HORTA, M. C.; MORAES-FILHO, J.; PACHECO, R. C.; LABRUNA, M. B.; AGUIAR, D. M. Seroprevalence and risk factors to Ehrlichia spp. and Rickettsia spp. in dogs from the Pantanal Region of Mato Grosso State, Brazil. Ticks and Tick-borne Diseases, Lippersdorf, v. 2, n. 4, p. 213-218, 2011.

MILAGRES, B. S.; PADILHA, A. F.; MONTANDON, C. E.; FREITAS, R. N.; PACHECO, R. C.; WALKER, D. H.; LABRUNA, M. B.; MAFRA, C. L.; GALVÃO, M. A. M. Spotted fever group rickettsia in small rodents from areas of low endemicity for brazilian spotted fever in the eastern region of Minas Gerais state, Brazil. The American Journal of Tropical Medicine and Hygiene, Deerfield, v. 88, n. 5, p. 937-939, 2013.

MORAES-FILHO, J.; HORTA, M. C.; PACHECO, R. D. C.; MAEDA, M. M.; GALANO, A.; OLIVEIRA, M. L. D.; YAI, L. E. O.; LABRUNA, M. B. Pesquisa de anticorpos anti-Rickettsia rickettsii em eqüinos do Centro de Controle de Zoonoses do município de São Paulo (CCZ/SP). Brazilian Journal of Veterinary Research and Animal Science, São Paulo, v. 46, n. 2, p. 85-91, 2009.

GOVERNO DE MATO GROSSO - MTGOV. Geografia de Mato Grosso. 2012. Disponível em: <http://www. mt.gov.br>. Acesso em: 01 dez. 2012.

PACHECO, R. C.; HORTA, M. C.; MORAES-FILHO, J.; ATALIBA, A. C.; PINTER, A.; LABRUNA, M. B. Rickettsial infection in capybaras (Hydrochoerus hydrochaeris) from São Paulo, Brazil: serological 
evidence for infection by Rickettsia bellii and Rickettsia parkeri. Biomédica, Bogotá, v. 27, n. 3, p. 364-371, 2007.

PACHECO, R. C.; HORTA, M. C.; PINTER, A.; MORAES-FILHO, J.; MARTINS, T. F.; NARDI, M. S.; SOUZA, S.; SOUZA, C. E.; SZABÓ, M. P. J.; RICHTZENHAIN, L. J. Pesquisa de Rickettsia spp. em carrapatos Amblyomma cajennense e Amblyomma dubitatum no estado de São Paulo. Revista da Sociedade Brasileira de Medicina Tropical, Uberaba, v. 42, n. 3, p. 351-353, 2009.

PACHECO, R. C.; MORAES-FILHO, J.; GUEDES, E.; SILVEIRA, I.; RICHTZENHAIN, L. J.; LEITE, R. C.; LABRUNA, M. B. Rickettsial infections of dogs, horses and ticks in Juiz de Fora, southeastern Brazil, and isolation of Rickettsia rickettsii from Rhipicephalus sanguineus ticks. Medical and Veterinary Entomology, Liverpool, v. 25, n. 2, p. 148-155, 2011.

PAROLA, P.; PADDOCK, C. D.; RAOULT, D. Tickborne rickettsioses around the world: emerging diseases challenging old concepts. Clinical Microbiology Reviews, Washington D.C., v. 18, n. 4, p. 719-756, 2005.

PENA, D. C. H.; MAFRA, C. L.; CALIC, S. B.; LABRUNA, M. B.; MILAGRES, B. S.; WALKER, D. H.; GALVÃO, M. A. M. Serologic survey for antibodies to Rickettsia among domestic and wild animal populations in Brazil. Clinical Microbiology and Infection, Basel, v. 15, n. 2, p. 243-244, 2009.

PHILIP, R.; CASPER, E.; ANACKER, R.; CORY, J.; HAYES, S.; BURGDORFER, W.; YUNKER, C. Rickettsia bellii sp. nov.: a tick-borne rickettsia, widely distributed in the United States, that is distinct from the spotted fever and typhus biogroups. International Journal of Systematic Bacteriology, Reading, v. 33, n. 1, p. 94-106, 1983.

PINTER, A.; LABRUNA, M. B. Isolation of Rickettsia rickettsii and Rickettsia bellii in cell culture from the tick Amblyomma aureolatum in Brazil. Annals of the New York Academy of Sciences, New York, v. 1078, n. 1, p. 523-529, 2006.

PINTER, A.; HORTA, M. C.; PACHECO, R. C.; MORAES-FILHO, J.; LABRUNA, M. B. Serosurvey of Rickettsia spp. in dogs and humans from an endemic area for Brazilian spotted fever in the State of São Paulo, Brazil. Cadernos de Saúde Pública, Rio de Janeiro, v. 24, n. 2, p. 247-252, 2008.

RAOULT, D.; ROUX, V. Rickettsioses as paradigms of new or emerging infectious diseases. Clinical Microbiology Reviews, Washington D.C., v. 10, n. 4, p. 694-719, 1997.

SANGIONI, L. A.; VOGEL, F. S. F.; CADORE, G.
C.; HILGER, R. B.; TONIM, R.; PACHECO, R. C.; OGRZEWALSKA, M.; LABRUNA, M. B. Rickettsial infection in Cerro Largo, State of Rio Grande do Sul, Brazil. Arquivo Brasileiro de Medicina Veterinária e Zootecnia, Belo Horizonte, v. 63, n. 2, p. 511-514, 2011.

SANGIONI, L. A.; HORTA, M. C.; VIANNA, M. C. B.; GENNARI, S. M.; SOARES, R. M.; GALVÃO, M. A. M.; SCHUMAKER, T. T. S.; FERREIRA, F.; VIDOTTO, O.; LABRUNA, M. B. Rickettsial infection in animals and brazilian spotted fever endemicity. Emerging Infectious Diseases, Atlanta, v. 11, p. 265-270, 2005.

SAITO, T. B.; CUNHA-FILHO, N. A.; PACHECO, R. C.; FERREIRA, F.; PAPPEN, F. G.; FARIAS, N. A. R.; LARSSON, C. E.; LABRUNA, M. B. Canine infection by Rickettsiae and Ehrlichiae in southern Brazil. The American Journal of Tropical Medicine and Hygiene, Deerfield, v. 79, n. 1, p. 102-108, 2008.

SILVA, M. E.; RIBEIRO, R. R.; COSTA, J. O.; MORAES-FILHO, J.; PACHECO, R. C.; LABRUNA, M. B. Prevalência de anticorpos anti-Rickettsia spp. em cães da cidade de Belo Horizonte, MG. Arquivo Brasileiro de Medicina Veterinária e Zootecnia, Belo Horizonte, v. 62, n. 4, p. 1007-1010, 2010.

SILVA, N.; EREMEEVA, M. E.; ROZENTAL, T.; RIBEIRO, G. S.; PADDOCK, C. D.; RAMOS, E. A. G.; FAVACHO, A. R. M.; REIS, M. G.; DASCH, G. A.; DE LEMOS, E. R. S. Eschar-associated spotted fever rickettsiosis, Bahia, Brazil. Emerging Infectious Diseases, Atlanta, v. 17, n. 2, p. 275, 2011.

SILVEIRA, I.; PACHECO, R. C.; SZABO, M. P.; RAMOS, H. G.; LABRUNA, M. B. Rickettsia parkeri in Brazil. Emerging Infectious Diseases, Atlanta, v. 13, n. 7, p. 1111-1113, 2007.

SOUZA, C. E.; MORAES-FILHO, J.; OGRZEWALSKA, M.; UCHOA, F. C.; HORTA, M. C.; SOUZA, S. S. L.; BORBA, R. C. M.; LABRUNA, M. B. Experimental infection of capybaras Hydrochoerus hydrochaeris by Rickettsia rickettsii and evaluation of the transmission of the infection to ticks Amblyomma cajennense. Veterinary Parasitology, Amsterdam, v. 161, n. 1-2, p. 116-121, 2009.

SPOLIDORIO, M. G.; LABRUNA, M. B.; MANTOVANI, E.; BRANDAO,P.E.; RICHTZENHAIN, L. J.; YOSHINARI, N. H. Novel Spotted Fever Group Rickettsiosis, Brazil. Emerging Infectious Diseases, Atlanta, v. 16, n. 3, p. 521-523, 2010a.

SPOLIDORIO, M. G.; LABRUNA, M. B.; MACHADO, R. Z.; MORAES-FILHO, J.; ZAGO, A. M.; DONATELE, D. M.; PINHEIRO, S. R.; SILVEIRA, I.; CALIARI, K. M.; YOSHINARI, N. H. Survey for tick-borne zoonoses 
in the state of Espírito Santo, southeastern Brazil. The American Journal of Tropical Medicine and Hygiene, Deerfield, v. 83, n. 1, p. 201-206, 2010b.

SPOLIDORIO, M. G.; MINERVINO, A. H. H.; VALADAS, S. Y. O. B.; SOARES, H. S.; NEVES, K. A. L.; LABRUNA, M. B.; RIBEIRO, M. F. B.; GENNARI, S. M. Serosurvey for tick-borne diseases in dogs from the Eastern Amazon, Brazil. Revista Brasileira de Parasitologia Veterinária, Jaboticabal, v. 22, n. 2, p. 214219, 2013.

TAMEKUNI, K.; TOLEDO, R. S.; SILVA FILHO, M. F.; HAYDU, V. B.; PACHECO, R. C.; LABRUNA, M. B.; DUMLER, J. S.; VIDOTTO, O. Survey of rickettsiae in humans, dogs, horses, and ticks in Northern Paraná, Brazil. Semina: Ciências Agrárias, Londrina, v. 32, n. 4, p. 1527-1538, 2011.
TOLEDO, R. S.; TAMEKUNI, K.; FILHO, M. F. S.; HAYDU, V. B.; BARBIERI, A. R. M.; HILTEL, A. C.; PACHECO, R. C.; LABRUNA, M. B.; DUMLER, J. S.; VIDOTTO, O. Infection by Spotted fever rickettsiae in people, dogs, horses and ticks in Londrina, Paraná state, Brazil. Zoonoses and Public Health, Berlin, v. 58, n. 6, p. 416-423, 2011.

VIANNA, M. C. B.; HORTA, M. C.; SANGIONI, L. A.; CORTEZ, A.; SOARES, R. M.; MAFRA, C. L.; GALVÃO, M. A. M.; LABRUNA, M. B.; GENNARI, S. M. Rickettsial spotted fever in capoeirao village, Itabira, Minas Gerais, Brazil. Revista do Instituto de Medicina Tropical de São Paulo, São Paulo, v. 50, n. 5, p. 297-301, 2008. 
